\section{Equivalências semântica e de itens da edição em português do Vécu et Santé Perçue de I'Adolescent: questionário de avaliação da qualidade de vida do adolescente}

\author{
Semantic and item equivalences of the Brazilian \\ Portuguese version of the Vécu et Santé \\ Perçue de l'Adolescent: an adolescent \\ quality-of-life questionnaire
}

\author{
${ }^{1}$ Universidade Federal do \\ Rio de Janeiro, Rio de Janeiro, \\ Brasil. \\ 2 Universidade do Estado do \\ Rio de Janeiro, Rio de Janeiro, \\ Brasil. \\ Correspondência \\ M. T. Aires \\ Universidade Federal do \\ Rio de Janeiro. \\ Rua Bruno Lobo 50, Rio de \\ Janeiro, $R J$ \\ 24360-020, Brasil. \\ marianataires@gmail.com
}

Abstract

In Brazil, no instruments evaluating adolescents' health-related quality of life (HRQoL) have been developed from the adolescents' point of view. The use of an instrument developed in another socio-cultural context requires prior cultural adaptation, including conceptual, item, and semantic equivalence in the process. Vécu et Santé Perçue de l'Adolescent (VSP-A) is a French generic instrument developed to evaluate adolescents' HRQoL. The objective of the study was to evaluate the semantic, item, and conceptual equivalence of a Brazilian Portuguese version of the VSP-A. VSP-A was translated into Portuguese, back-translated (with steps evaluated by experts), and pre-tested in a group of 14 adolescents. Discussion with experts resulted in a final version of the questionnaire, considered semantically and conceptually equivalent to the original, despite the need to modify some items to facilitate use. Pretest results showed that the instrument is easy for adolescents to understand and answer. For use in Brazil, its psychometric properties need to be evaluated.

Adolescent; Quality of Life; Validation Studies; Questionnaires
Mariana Tschoepke Aires 1

Guilherme Loureiro Werneck 2

\section{Introdução}

O Brasil carece de instrumentos genéricos para determinar a qualidade de vida relacionada à saúde de adolescentes, desfecho útil na avaliação de intervenções terapêuticas e programas de promoção da saúde. Vários instrumentos genéricos estão disponíveis na literatura, destacando-se os seguintes com versões específicas para adolescentes (10-18 anos), abordando qualidade de vida relacionada à saúde como um construto multidimensional: German Quality of Life Questionnaire (KINDL); Pediatric Quality of Life Inventory (PedsQL); Kidscreen e Vécu et Santé Perçue de l'Adolescent (VSP-A) 1. Até o momento, há versões para uso no Brasil do PedsQL 2 e Kisdscreen ${ }^{3}$. KINDL, PedsQL, Kidscreen e VSP-A apresentam concepções sobre qualidade de vida relacionada à saúde e propriedades psicométricas similares, mas VSP-A apresenta peculiaridades que justificam sua escolha.

OVSP-A foi desenvolvido para investigar qualidade de vida relacionada à saúde de adolescentes saudáveis ou doentes, com base em entrevistas e grupos focais, fundamentado no conceito de qualidade de vida como um construto multidimensional a ser apreendido a partir da percepção dos próprios adolescentes sobre sua vida e saúde 4,5. As dimensões do VSP-A contemplam aspectos relevantes para adolescência, período marcado por profundas mudanças nos relacionamentos interpessoais, como menor partici- 
pação dos pais nas atividades diárias e maior intimidade nos relacionamentos com pares da mesma idade. VSP-A é o único desses instrumentos que inclui domínios relacionados à vida sexual, aspecto relevante para a qualidade de vida relacionada à saúde nessa faixa etária (Tabela 1). É também o único originalmente desenvolvido em língua latina (francês), o que pode contribuir para maior identidade cultural do construto de qualidade de vida relacionada à saúde entre a população na qual o instrumento foi desenvolvido e os adolescentes brasileiros.

VSP-A foi adaptado para Espanha e Colômbia mostrando boa equivalência conceitual, de itens e semântica, após discussão com especialistas e adolescentes 6,7,8. Na Espanha, mostrou boa validade (comparando-se com outro instrumento de qualidade de vida relacionada à saúde) e confiabilidade (consistência interna/reprodutibilidade) 6,8 .

Instrumentos elaborados para avaliar qualidade de vida relacionada à saúde, antes de aplicados em outro meio, devem ser submetidos a processo de adaptação transcultural a fim de se obter medidas válidas e confiáveis, comparáveis às do instrumento original 9 . O objetivo deste estudo é verificar as equivalências conceitual, de itens e semântica da edição em português do VSP-A, etapas iniciais do processo de adaptação transcultural para seu uso em adolescentes brasileiros.

\section{Métodos}

A metodologia adotada, discutida previamente com os autores do VSP-A, compreendeu cinco etapas: tradução, retrotradução, avaliação de equivalência semântica, discussão com especialistas e pré-teste.

VSP-A, anônimo e autopreenchível, contempla dez dimensões, possuindo 36 itens com cinco opções de respostas (Tabelas 2 e 3) 4,5. A validade de construto foi investigada utilizando-se análise de fatores, que gerou dez fatores coerentes com as dimensões originalmente propostas 4,5 . Os itens apresentaram boa validade convergente (cada um correlacionando-se fortemente com sua dimensão) e discriminante (itens correlacionandose mais com sua dimensão do que com outras). A validade externa foi confirmada mostrando que meninas e adolescentes mais velhos apresentaram escores mais baixos, em acordo com a literatura. A consistência interna das escalas variou de 0,76 a 0,86 e a confiabilidade teste-reteste de 0,6 a 0,7 4,5. É um instrumento aceitável (dados faltantes $<5 \%$, pouco efeito piso/teto) e útil para discriminar entre adolescentes saudáveis e doentes 4,5 . Escore de dois itens da dimensão escolar e escore do bem-estar psicológico foram calculados em reverso, de forma que escores mais altos indicam melhor qualidade de vida relacionada à saúde. Os escores das dimensões e do VSP-A variam de 0 (pior qualidade de vida relacionada à saúde)

Números de itens e dimensões de instrumentos genéricos e multidimensionais de avaliação de qualidade de vida relacionada à saúde de crianças e adolescentes disponíveis na literatura.

\begin{tabular}{|c|c|c|c|c|}
\hline Instrumento & Origem & Edições & Itens & Número e descrição das dimensões \\
\hline $\begin{array}{l}\text { Pediatric Quality of Life Inventory } \\
\text { (PedsQoL) - módulo genérico * }\end{array}$ & $\begin{array}{l}\text { Estados } \\
\text { Unidos }\end{array}$ & $\begin{array}{l}\text { Para crianças e } \\
\text { adolescentes }\end{array}$ & 23 & $\begin{array}{l}4 \text { dimensões: bem-estar físico; bem-estar emocional; bem-estar } \\
\text { social; desempenho escolar }\end{array}$ \\
\hline Kidscreen $* \star$ & Europa & $\begin{array}{l}\text { Para crianças e } \\
\text { adolescentes }\end{array}$ & 52 & $\begin{array}{l}10 \text { dimensões: saúde e atividade física; sentimentos; estado } \\
\text { emocional; autopercepção; autonomia e tempo livre; família/ } \\
\text { ambiente familiar; aspecto financeiro; amigos e apoio social; } \\
\text { ambiente escolar; provocação/bullying }\end{array}$ \\
\hline $\begin{array}{l}\text { German Quality of Life } \\
\text { Questionnaire (KINDL) *** }\end{array}$ & Alemanha & $\begin{array}{l}\text { Para crianças e } \\
\text { adolescentes }\end{array}$ & 24 & $\begin{array}{l}6 \text { dimensões: bem-estar físico; bem-estar emocional; autoestima; } \\
\text { família; amigos; funcionamento no dia a dia }\end{array}$ \\
\hline $\begin{array}{l}\text { Vécu et Santé Perçue de } \\
\text { l'Adolescent (VSP-A) \# }\end{array}$ & França & $\begin{array}{l}\text { Para } \\
\text { adolescentes }\end{array}$ & 36 & $\begin{array}{l}10 \text { dimensões bem-estar físico; bem-estar psicológico; } \\
\text { relacionamento com os pais; relacionamento com os amigos; } \\
\text { relacionamento com os professores; desempenho escolar; imagem } \\
\text { corporal; vida sexual e sentimental; atividades de lazer; vitalidade }\end{array}$ \\
\hline
\end{tabular}

\footnotetext{
* PedsQol: módulo genérico e módulo de impacto familiar foram adaptados para uso no Brasil;

** Kidscreen: as versões para pais/tutores e crianças/adolescentes foram adaptadas para uso no Brasil;

*** KINDL: apresenta versões que podem ser respondidas por pais, crianças e adolescentes;

\# VSP-A: desenvolvido exclusivamente para adolescentes com base na sua percepção de saúde.
} 
Itens e dimensões que compõem o Vécu et Santé Perçue de l'Adolescent (VSP-A), aplicáveis ao nosso meio após discussão com especialistas em pediatria ، hebiatria e saúde pública.

\begin{tabular}{|c|c|}
\hline Dimensões & Itens \\
\hline \multirow[t]{5}{*}{ Bem-estar psicológico } & Sentiu-se inquieto(a), preocupado(a) \\
\hline & Sentiu-se triste, deprimido(a) \\
\hline & Sentiu-se estressado(a) \\
\hline & Sentiu-se facilmente desanimado(a) \\
\hline & Sentiu-se angustiado(a) ou com medo ao pensar no futuro \\
\hline \multirow[t]{4}{*}{ Atividades de lazer } & Encontrar-se em grupo com seus amigos e amigas \\
\hline & Sair (passear na rua, fazer compras, ir à piscina, ao cinema, ir ao shopping) \\
\hline & Ir para a casa de seus amigos/ suas amigas \\
\hline & Sair ao ar livre para se divertir com seus amigos e amigas (andar a pé, de bicicleta, jogar futebol ou vôlei) \\
\hline \multirow[t]{5}{*}{ Relacionamento com os amigos } & Conversar com seus amigos/suas amigas, seus colegas \\
\hline & Abrir-se, falar de seus problemas com seus amigos, suas amigas, seus colegas \\
\hline & Expressar-se livremente, dar sua opinião a seus amigos/suas amigas, seus colegas \\
\hline & Sentiu-se apoiado(a), ajudado(a) por seus amigos/suas amigas, seus colegas \\
\hline & Sentiu-se compreendido(a), tranquilizado(a) por seus amigos/suas amigas, seus colegas \\
\hline \multirow[t]{4}{*}{ Relacionamento com os pais } & Abrir-se, falar de seus problemas com seus pais \\
\hline & Expressar-se livremente, dar sua opinião a seus pais \\
\hline & Sentiu-se compreendido(a), tranquilizado(a) por seus pais \\
\hline & Receber conselhos dos pais \\
\hline \multirow[t]{3}{*}{ Relacionamento com os professores } & Sentiu-se ajudado(a) por seus professores \\
\hline & Sentiu-se compreendido(a) por seus professores \\
\hline & Sentiu-se aceito(a), respeitado(a) por seus professores \\
\hline \multirow[t]{2}{*}{ Desempenho escolar } & Sentiu-se satisfeito(a) com suas notas na escola \\
\hline & Bons resultados na escola \\
\hline \multirow[t]{2}{*}{ Imagem corporal } & Complexado(a) com seu físico, sua aparência \\
\hline & Gordo(a) ou magro(a) demais, alto(a) ou baixo(a) demais \\
\hline \multirow[t]{4}{*}{ Bem estar físico } & Sentiu-se sem energia \\
\hline & Sentiu-se em boa forma física \\
\hline & Sentiu-se fraco(a), cansado(a) \\
\hline & Sentiu dores, mal-estar em alguma parte do corpo \\
\hline \multirow[t]{5}{*}{ Vitalidade } & Sentiu que teve ânimo \\
\hline & Estava disposto(a) a ver o lado bom da vida \\
\hline & Tudo ia bem à sua volta \\
\hline & Confiante em si mesmo(a) \\
\hline & Contente, satisfeito(a) com sua vida \\
\hline \multirow[t]{2}{*}{ Vida sexual e sentimental } & Satisfeito(a) em sua vida sentimental com seu namorado/sua namorada \\
\hline & Satisfeito(a) com sua vida sexual \\
\hline
\end{tabular}

a 100 (melhor qualidade de vida relacionada à saúde). A pontuação global é obtida como média de todos os itens 4,5 .

\section{Avaliação das equivalências conceitual e de itens}

Realizada presencialmente por dois pediatras, um hebiatra e dois especialistas em saúde pública, experientes na adaptação transcultural de instrumentos de qualidade de vida relacionada à saúde e vivência clínica com adolescentes, que analisaram as dimensões que compõem o instrumento e a capacidade dos itens em refleti-las.

\section{Avaliação da equivalência semântica}

O VSP-A foi traduzido para o português por tradutor juramentado fluente em português e francês (idioma materno: português). A edição em 
Avaliação dos significados geral e referencial, comparando-se o instrumento original e a tradução para o português e o original e a retradução para o francês.

\begin{tabular}{|c|c|c|c|c|}
\hline Original & Tradução & Retradução & $\begin{array}{l}\text { Avaliação do } \\
\text { significado geral } \\
\text { (entre original e } \\
\text { tradução) }\end{array}$ & $\begin{array}{l}\text { Avaliação do } \\
\text { significado } \\
\text { referencial } \\
\text { (entre original e } \\
\text { retradução) [\%] }\end{array}$ \\
\hline $\begin{array}{l}\text { Vécu et Santé Perçue des } \\
\text { Adolescents }\end{array}$ & $\begin{array}{l}\text { Vivência e Saúde Percebida do } \\
\text { Adolescente }\end{array}$ & $\begin{array}{l}\text { Expérience et Santé Observée } \\
\text { de l'Adolescent }\end{array}$ & Inalterado & 80 \\
\hline $\begin{array}{l}\text { Ce questionnaire, construit à partir } \\
\text { de l'opinion d'adolescents, vise à } \\
\text { évaluer les conséquences de votre } \\
\text { santé sur votre vie de tous les jours }\end{array}$ & $\begin{array}{l}\text { Este questionário, construído } \\
\text { com base na opinião de } \\
\text { adolescentes, tem por objetivo } \\
\text { avaliar as consequências de sua } \\
\text { saúde sobre sua vida cotidiana }\end{array}$ & $\begin{array}{l}\text { Ce questionnaire, réalisé } \\
\text { à partir de l'opinion des } \\
\text { adolescents, a pour objectif } \\
\text { d'évaluer les conséquences } \\
\text { de votre santé sur votre vie } \\
\text { quotidienne }\end{array}$ & Inalterado & 100 \\
\hline $\begin{array}{l}\text { Merci de bien vouloir répondre } \\
\text { à chaque question en cochant la } \\
\text { case que correspond le plus à ce } \\
\text { que vous avez pense ou ressenti } \\
\text { au cours de ces quatre dernières } \\
\text { semaines. Il n'y a pas de bonnes } \\
\text { ou de mauvaises réponses. Si } \\
\text { certaines questions ne vous } \\
\text { concernent pas, n'y répondez pas } \\
\text { mais entourez le numéro de la } \\
\text { question }\end{array}$ & $\begin{array}{l}\text { Agradecemos se puder } \\
\text { responder a cada pergunta } \\
\text { marcando a caixinha que } \\
\text { mais corresponde ao que } \\
\text { você pensou ou sentiu nessas } \\
\text { quatro últimas semanas. } \\
\text { Não há respostas certas ou } \\
\text { erradas. Se algumas perguntas } \\
\text { não lhe disserem respeito, } \\
\text { não responda, mas faça um } \\
\text { pequeno círculo em volta do } \\
\text { número da pergunta }\end{array}$ & $\begin{array}{l}\text { Nous vous remercions de bien } \\
\text { vouloir répondre à chaque } \\
\text { question en cochant la case qui } \\
\text { correspond le mieux à ce que } \\
\text { vous avez pensé ou ressenti ces } \\
\text { quatre dernières semaines. Il } \\
\text { n'y a pas de réponses vraies ou } \\
\text { fausses. Si certaines questions } \\
\text { ne s'appliquent pas à vous, ne } \\
\text { répondez pas, mais entourez le } \\
\text { numéro de la question }\end{array}$ & Inalterado & 90 \\
\hline Merci de votre participation! & $\begin{array}{l}\text { Agradecemos muito a sua } \\
\text { participação! }\end{array}$ & $\begin{array}{l}\text { En vous remerciant de votre } \\
\text { participation! }\end{array}$ & Inalterado & 100 \\
\hline $\begin{array}{l}\text { Durant les quatre dernières } \\
\text { semaines avez-vous pu: }\end{array}$ & $\begin{array}{l}\text { Nas últimas quatro semanas, } \\
\text { você pôde: }\end{array}$ & $\begin{array}{l}\text { Pendant les quatre dernières } \\
\text { semaines, vous avez pu: }\end{array}$ & Inalterado & 100 \\
\hline $\begin{array}{l}\text { Vous retrouver en bande avec } \\
\text { vos copains, vos copines? }\end{array}$ & $\begin{array}{l}\text { Encontrar em grupo com seus } \\
\text { amigos e amigas? }\end{array}$ & $\begin{array}{l}\text { Rencontrer vos amis(es) en } \\
\text { groupe? }\end{array}$ & Inalterado & 90 \\
\hline $\begin{array}{l}\text { Sortir (aller en ville, faire des } \\
\text { achats, aller à la piscine, au } \\
\text { cinéma...)? }\end{array}$ & $\begin{array}{l}\text { Sair (passear na rua, fazer } \\
\text { compras, ir à piscina, ao } \\
\text { cinema)? }\end{array}$ & $\begin{array}{l}\text { Sortir (vous promener dans la } \\
\text { rue, faire des courses, aller à la } \\
\text { piscine, au cinéma)? }\end{array}$ & Inalterado & 90 \\
\hline $\begin{array}{l}\text { Discuter avec vos copains, vos } \\
\text { copines? }\end{array}$ & $\begin{array}{l}\text { Conversar com seus amigos/ } \\
\text { suas amigas? }\end{array}$ & Bavarder avec vos amis(es)? & Inalterado & 80 \\
\hline $\begin{array}{l}\text { Vous confier, parler de vos } \\
\text { problèmes avec vos copains, } \\
\text { vos copines? }\end{array}$ & $\begin{array}{l}\text { Abrir-se, falar de seus } \\
\text { problemas com seus amigos, } \\
\text { suas amigas? }\end{array}$ & $\begin{array}{l}\text { Vous ouvrir, parler de vos } \\
\text { problèmes avec vos amis(es)? }\end{array}$ & Inalterado & 80 \\
\hline $\begin{array}{l}\text { Vous exprimer librement, } \\
\text { donner votre avis à vos copains, } \\
\text { vos copines? }\end{array}$ & $\begin{array}{l}\text { Expressar-se livremente, dar } \\
\text { sua opinião a seus amigos/suas } \\
\text { amigas? }\end{array}$ & $\begin{array}{l}\text { Vous exprimer librement, } \\
\text { donner votre opinion à vos } \\
\text { amis(es)? }\end{array}$ & Inalterado & 100 \\
\hline $\begin{array}{l}\text { Vous confier, parler de vos } \\
\text { problèmesavec vos parents? }\end{array}$ & $\begin{array}{l}\text { Abrir-se, falar de seus } \\
\text { problemas com seus pais? }\end{array}$ & $\begin{array}{l}\text { Vous ouvrir, parler de vos } \\
\text { problèmes avec vos parents? }\end{array}$ & Inalterado & 100 \\
\hline $\begin{array}{l}\text { Vous exprimer librement, } \\
\text { donner votre avis à vos parents? }\end{array}$ & $\begin{array}{l}\text { Expressar-se livremente, dar sua } \\
\text { opinião a seus pais? }\end{array}$ & $\begin{array}{l}\text { Vous exprimer librement, } \\
\text { donner votre opinion à vos } \\
\text { parents? }\end{array}$ & Inalterado & 100 \\
\hline
\end{tabular}

(continua) 


\begin{tabular}{|c|c|c|c|c|}
\hline Original & Tradução & Retradução & $\begin{array}{l}\text { Avaliação do } \\
\text { significado geral } \\
\text { (entre original e } \\
\text { tradução) }\end{array}$ & $\begin{array}{l}\text { Avaliação do } \\
\text { significado } \\
\text { referencial } \\
\text { (entre original } \\
\text { retradução) [\%] }\end{array}$ \\
\hline $\begin{array}{l}\text { Durant les quatre dernières } \\
\text { semaines: }\end{array}$ & $\begin{array}{l}\text { Nas últimas quatro semanas, } \\
\text { você: }\end{array}$ & $\begin{array}{l}\text { Pendant les quatre dernières } \\
\text { semaines, vous: }\end{array}$ & Inalterado & 100 \\
\hline $\begin{array}{l}\text { Etes-vous allé(e) chez vos } \\
\text { copains, vos copines? }\end{array}$ & $\begin{array}{l}\text { Foi para a casa de seus amigos/ } \\
\text { suas amigas? }\end{array}$ & Etes allez chez vos amis(es)? & Inalterado & 100 \\
\hline $\begin{array}{l}\text { Etes-vous allé(e) jouer dehors } \\
\text { avec vos copains, vos copines } \\
\text { (vélo, foot, roller...)? }\end{array}$ & $\begin{array}{l}\text { Saiu ao ar livre para se divertir } \\
\text { com seus amigos e amigas } \\
\text { (andar a pé, de bicicleta, } \\
\text { de skate)? }\end{array}$ & $\begin{array}{l}\text { Etes sorti en plein air pour } \\
\text { vous amuser avec vos amis(es) } \\
\text { (promenade à pied, en } \\
\text { bicyclette, en skateboard)? }\end{array}$ & Pouco alterado & 100 \\
\hline $\begin{array}{l}\text { Durant les quatre dernières } \\
\text { semaines avez-vous étè: }\end{array}$ & $\begin{array}{l}\text { Nas últimas quatro semanas, } \\
\text { sentiu-se: }\end{array}$ & $\begin{array}{l}\text { Pendant les quatre dernières } \\
\text { semaines, vous vous êtes senti: }\end{array}$ & Inalterado & 100 \\
\hline Inquiet(e), soucieux(se)? & Inquieto(a), preocupado(a)? & Inquiet(e), soucieux(se)? & Inalterado & 100 \\
\hline Triste, déprimé(e)? & Triste, deprimido(a)? & Triste, déprimé(e)? & Inalterado & 100 \\
\hline Stressé(e)? & Estressado(a)? & Stressé(e)? & Inalterado & 100 \\
\hline Facilement découragé(e)? & Facilmente desanimado(a)? & Facilement découragé(e)? & Inalterado & 100 \\
\hline $\begin{array}{l}\text { Angoissé(e) ou eu peur en } \\
\text { pensant à l'avenir? }\end{array}$ & $\begin{array}{l}\text { Angustiado(a) ou com medo ao } \\
\text { pensar no futuro? }\end{array}$ & $\begin{array}{l}\text { Angoissé(e), ou apeuré(e) en } \\
\text { pensant à l'avenir? }\end{array}$ & Inalterado & 100 \\
\hline $\begin{array}{l}\text { Content(e), satisfait(e) de votre } \\
\text { vie? }\end{array}$ & $\begin{array}{l}\text { Contente, satisfeito(a) com sua } \\
\text { vida? }\end{array}$ & $\begin{array}{l}\text { Content(e), satisfait(e) de votre } \\
\text { vie? }\end{array}$ & Inalterado & 100 \\
\hline $\begin{array}{l}\text { Entouré(e), aidé(e) par vos } \\
\text { copains, vos copines? }\end{array}$ & $\begin{array}{l}\text { Apoiado(a), ajudado(a) por seus } \\
\text { amigos/suas amigas? }\end{array}$ & $\begin{array}{l}\text { Soutenu(e), aidé(e) par vos } \\
\text { amis(es)? }\end{array}$ & Pouco alterado & 100 \\
\hline $\begin{array}{l}\text { Compris(e), rassuré(e) par vos } \\
\text { copains, vos copines? }\end{array}$ & $\begin{array}{l}\text { Compreendido(a), } \\
\text { tranquilizado(a) por seus } \\
\text { amigos/suas amigas? }\end{array}$ & $\begin{array}{l}\text { Compris(e), rassuré(e) par vos } \\
\text { amis(es)? }\end{array}$ & Pouco alterado & 100 \\
\hline $\begin{array}{l}\text { Satisfait de votre vie } \\
\text { sentimentale avec votre petit(e) } \\
\text { ami(e)? }\end{array}$ & $\begin{array}{l}\text { Satisfeito(a) em sua vida } \\
\text { sentimental com seu } \\
\text { namorado/sua namorada? }\end{array}$ & $\begin{array}{l}\text { Satisfait(e) de votre vie } \\
\text { sentimentale avec votre copain/ } \\
\text { votre copine? }\end{array}$ & Inalterado & 100 \\
\hline Satisfait de votre vie sexuelle? & $\begin{array}{l}\text { Satisfeito(a) com sua vida } \\
\text { sexual? }\end{array}$ & $\begin{array}{l}\text { Satisfait(e) de votre vie } \\
\text { sexuelle? }\end{array}$ & Inalterado & 100 \\
\hline $\begin{array}{l}\text { Compris(e), rassuré(e) par vos } \\
\text { parents? }\end{array}$ & $\begin{array}{l}\text { Compreendido(a), } \\
\text { tranquilizado(a) por seus pais? }\end{array}$ & $\begin{array}{l}\text { Compris(e), rassuré(e) par vos } \\
\text { parents? }\end{array}$ & Pouco alterado & 100 \\
\hline $\begin{array}{l}\text { Content(e) de vos résultats } \\
\text { scolaires? }\end{array}$ & $\begin{array}{l}\text { Satisfeito(a) com seus } \\
\text { resultados escolares? }\end{array}$ & $\begin{array}{l}\text { Satisfait(e) de vos résultats } \\
\text { scolaires? }\end{array}$ & Inalterado & 100 \\
\hline Aidé(e) par vos professeurs? & $\begin{array}{l}\text { Ajudado(a) por seus } \\
\text { professores? }\end{array}$ & Aidé(e) par vos professeurs? & Inalterado & 100 \\
\hline Compris(e) par vos professeurs? & $\begin{array}{l}\text { Compreendido(a) por seus } \\
\text { professores? }\end{array}$ & Compris(e) par vos professeurs? & Inalterado & 100 \\
\hline $\begin{array}{l}\text { Accepté(e), respecté(e) par vos } \\
\text { professeurs? }\end{array}$ & $\begin{array}{l}\text { Aceito(a), respeitado(a) por } \\
\text { seus professores? }\end{array}$ & $\begin{array}{l}\text { Accepté(e), respecté(e) par vos } \\
\text { professeurs? }\end{array}$ & Inalterado & 100 \\
\hline $\begin{array}{l}\text { Durant les quatre dernières } \\
\text { semaines vous êtes-vous senti(e): }\end{array}$ & $\begin{array}{l}\text { Nas últimas quatro semanas, } \\
\text { sentiu-se: }\end{array}$ & $\begin{array}{l}\text { Pendant les quatre dernières } \\
\text { semaines, vous vous êtes senti: }\end{array}$ & Inalterado & 100 \\
\hline $\begin{array}{l}\text { Complexé(e) par votre physique, } \\
\text { par votre apparence? }\end{array}$ & $\begin{array}{l}\text { Complexado(a) com seu físico, } \\
\text { sua aparência? }\end{array}$ & $\begin{array}{l}\text { Complexé(e) par votre } \\
\text { physique, votre apparence? }\end{array}$ & Inalterado & 100 \\
\hline $\begin{array}{l}\text { Trop gros(se) ou trop maigre, } \\
\text { trop grand(e) ou trop petit(e)? }\end{array}$ & $\begin{array}{l}\text { Gordo(a) ou magro(a) demais, } \\
\text { alto(a) ou baixo(a) demais? }\end{array}$ & $\begin{array}{l}\text { Trop gros(se) ou trop maigre, } \\
\text { trop grand(e) ou trop petit(e)? }\end{array}$ & Inalterado & 100 \\
\hline
\end{tabular}

(continua) 


\begin{tabular}{|c|c|c|c|c|}
\hline Original & Tradução & Retradução & $\begin{array}{c}\text { Avaliação do } \\
\text { significado geral } \\
\text { (entre original e } \\
\text { tradução) }\end{array}$ & $\begin{array}{l}\text { Avaliação do } \\
\text { significado } \\
\text { referencial } \\
\text { (entre original } \\
\text { retradução) [\%] }\end{array}$ \\
\hline $\begin{array}{l}\text { Durant les quatre dernières } \\
\text { semaines: }\end{array}$ & Nas últimas quatro semanas: & $\begin{array}{l}\text { Pendant les quatre dernières } \\
\text { semaines, vous vous êtes senti: }\end{array}$ & Inalterado & 100 \\
\hline Avez-vous manqué d'énergie? & Sentiu-se sem energia? & Sans énergie? & Inalterado & 100 \\
\hline $\begin{array}{l}\text { Avez-vous été en bonne forme } \\
\text { physique? }\end{array}$ & Sentiu-se em boa forma física? & En pleine forme physique? & Inalterado & 90 \\
\hline $\begin{array}{l}\text { Vous êtes-vous senti(e) } \\
\text { faible,fatigué(e)? }\end{array}$ & Sentiu-se fraco(a), cansado(a)? & Faible, fatigué(e)? & Inalterado & 100 \\
\hline $\begin{array}{l}\text { Vos parents vous ont-ils donné } \\
\text { des conseils? }\end{array}$ & Seus pais the deram conselhos? & $\begin{array}{l}\text { Vos parents vous ont donné des } \\
\text { conseils? }\end{array}$ & Inalterado & 100 \\
\hline $\begin{array}{l}\text { Durant les quatre dernières } \\
\text { semaines avez-vous eu: }\end{array}$ & $\begin{array}{l}\text { Nas últimas quatro semanas, } \\
\text { sentiu: }\end{array}$ & $\begin{array}{l}\text { Pendant les quatre dernières } \\
\text { semaines, vous avez senti: }\end{array}$ & Inalterado & 100 \\
\hline Le moral? & Que estava animado(a)? & $\begin{array}{l}\text { Que vous étiez en pleine } \\
\text { forme? }\end{array}$ & Inalterado & 0 \\
\hline $\begin{array}{l}\text { Tendance à prendre la vie du } \\
\text { bon côté? }\end{array}$ & $\begin{array}{l}\text { Que estava inclinado(a) a ver o } \\
\text { lado bom da vida? }\end{array}$ & $\begin{array}{l}\text { Que vous aviez tendance à voir } \\
\text { plutôt le bon côté de la vie? }\end{array}$ & Inalterado & 100 \\
\hline $\begin{array}{l}L^{\prime} \text { impression que tout allait bien } \\
\text { autour de vous? }\end{array}$ & Que tudo ia bem à sua volta? & $\begin{array}{l}\text { Que tout allait bien autour de } \\
\text { vous? }\end{array}$ & Inalterado & 100 \\
\hline $\begin{array}{l}\text { Confiance en vous, été sûr(e) } \\
\text { de vous? }\end{array}$ & Confiança em si mesmo(a)? & Confiance en vous-même? & Inalterado & 100 \\
\hline $\begin{array}{l}\text { De bons résultats au collège, } \\
\text { au lycée? }\end{array}$ & $\begin{array}{l}\text { Que teve bons resultados na } \\
\text { escola? }\end{array}$ & $\begin{array}{l}\text { Que vous avez obtenu des } \\
\text { bons résultats scolaires? }\end{array}$ & Inalterado & 100 \\
\hline Des douleurs, mal quelque part? & $\begin{array}{l}\text { Dores, mal-estar em alguma } \\
\text { parte do corpo? }\end{array}$ & $\begin{array}{l}\text { Douleurs, trouble dans une } \\
\text { partie de votre corps? }\end{array}$ & Inalterado & 100 \\
\hline Jamais/Pas du tout & Nunca & Jamais & Inalterado & 100 \\
\hline Rarement/Un peu & Raramente/Um pouco & Raremen/Un peu & Inalterado & 100 \\
\hline Parfois/Moyennement & Às vezes/Médio & Quelquefois/Moyennement & Inalterado & 100 \\
\hline Souvent/Beaucoup & Frequentemente/Muito & Très souvent/Beaucoup & Inalterado & 100 \\
\hline Toujours/Enormément & Sempre/Imensamente & Toujours/Constamment & Inalterado & 100 \\
\hline $\begin{array}{l}\text { Remarques éventuelles et } \\
\text { informations complémentaires }\end{array}$ & $\begin{array}{l}\text { Eventuais comentários e } \\
\text { informações adicionais }\end{array}$ & $\begin{array}{l}\text { Autres commentaires et } \\
\text { informations complémentaires }\end{array}$ & Inalterado & 100 \\
\hline
\end{tabular}

português foi retraduzida para o francês por outro tradutor juramentado (idioma materno: francês), sem conhecimento do instrumento original.

As edições em português e francês foram confrontadas para avaliar a correspondência entre os significados geral e referencial dos itens. A equivalência com ênfase no significado geral foi avaliada por pediatra fluente em francês e português, confrontando-se o instrumento original e a tradução para português (avaliação: inalterado, pouco alterado, muito alterado e completamente alterado). Outro pediatra, também fluente em francês e português, avaliou o significado referencial dos itens comparando o instrumento original e a retradução para o francês utilizando escalas análogas visuais, posteriormente interpretadas em percentuais.

Três pediatras e dois especialistas em saúde pública discutiram os resultados das etapas anteriores. As questões não resolvidas foram levadas aos autores do instrumento original. Atenção especial foi dada ao vocabulário, sua adequação à idade, origem e nível educacional dos participantes.

\section{Pré-teste}

Quatorze adolescentes do nono ano de escola pública do Município de São Gonçalo, Rio de Janeiro, responderam anonimamente à edição 
proposta em português do VSP-A. Essa escola estava inserida em programa de saúde escolar; os alunos participavam, há 4 anos, de atividades de promoção da saúde sobre higiene, autocuidado, sexualidade e prevenção de acidentes. Os jovens foram orientados a fazer sugestões/críticas/observações sobre o instrumento. Para avaliar a aceitabilidade foi aferido o tempo de preenchimento e analisadas as respostas abertas.

Os participantes assinaram um termo de consentimento livre e esclarecido. O estudo foi aprovado pelo Comitê de Ética em Pesquisa do Instituto de Medicina Social da Universidade do Estado do Rio de Janeiro.

\section{Resultados}

Os especialistas concordaram que itens e dimensões doVSP-A são relevantes e aplicáveis ao nosso meio. Na apreciação da equivalência semântica (significado geral) nenhum item foi considerado muito ou completamente alterado. Na avaliação da equivalência semântica concernente ao significado referencial, observou-se equivalência superior a $80 \%$, excetuando-se o item "avez-vous eu le moral?", traduzido como "estava animado/a?" e retraduzido para "étiez en pleine forme?" (Tabela 3). Na proposição final, esse item ficou como “teve ânimo?", termo mais empregado no nosso meio.

Foram acrescentadas atividades como "ir ao shopping" e "jogar futebol e vôlei" nos itens referentes a lazer/diversão. Nos itens que avaliam relacionamento com amigos, acrescentamos o termo "colegas", utilizado entre nós.

No item referente ao desempenho escolar, a tradução foi "resultados escolares", modificada para "notas na escola”. No item que avalia vitalidade, a tradução foi "estava inclinado(a) a ver o lado bom da vida?", modificada para "estava disposto a ver o lado bom da vida?".

Nos itens concernentes ao relacionamento com pais, os especialistas sugeriram a substituição do termo "pais" por "responsáveis", pois comumente o lar fica sob a responsabilidade da mãe ou outro adulto. Essa questão foi levada aos autores do questionário original que orientaram manter o termo "pais", pois ao final do questionário os jovens poderiam informar quem são os seus responsáveis.

Participaram do pré-teste 14 alunos, pertencentes à classe social pouco favorecida, com idades entre 13 e 17 anos (média 14,1 anos), 57\% sexo feminino. Dois consideraram longo; todos acharam de fácil compreensão, contendo itens pertinentes. O tempo de preenchimento foi de 15 minutos, em média.

\section{Discussão}

Instrumentos válidos e confiáveis sobre qualidade de vida relacionada à saúde de adolescentes são necessários em nosso meio, motivando a adaptação do VSP-A. A apreciação das equivalências conceitual, de itens e semântica é pré-requisito para obtenção de ferramenta válida e confiável, permitindo comparações internacionais 9 .

Discussões com especialistas mostraram que os itens do VSP-A são aplicáveis ao nosso meio e captam conceitos importantes na adolescência. Observou-se que a grande maioria dos itens apresentava boa equivalência semântica. O item "avez-vous eu le moral?" também mostrou dificuldades no processo de adaptação do VSP-A na Colômbia. Nesse país, como no presente trabalho, foram inseridos exemplos no item relativo às atividades ao ar livre. Na Espanha e Colômbia, os itens foram discutidos em entrevistas cognitivas com adolescentes; a dimensão que gerou maior dificuldade foi "vida sexual/sentimental". Os jovens propuseram inserir como opção de resposta "não tenho", aceita pelos autores em ambos os países 6,7,8. Entre nós, como não houve esse tipo de discussão, tal questão não foi levantada.

Uma limitação deste estudo é que os adolescentes não participaram da apreciação da equivalência conceitual e de itens. Discussões com os adolescentes poderiam acrescentar muito à interpretação dos itens/conceitos do VSP-A e contribuir para versão em português mais adequada. Outra limitação foi a impossibilidade de realizar duas traduções e retraduções, como preconizado. Entretanto, as apreciações satisfatórias dos processos de tradução e retradução, os resultados favoráveis do pré-teste e o aval dos especialistas devem ter contribuído para minimizar esses problemas.

No presente estudo foram obtidas com sucesso as equivalências conceitual, de itens e semântica do VSP-A. As equivalências de mensuração, operacional e funcional estão descritas em outra publicação 10 . 


\section{Resumo}

No Brasil, não há instrumentos genéricos de avaliação da qualidade de vida relacionada à saúde em adolescentes desenvolvidos com base em seus pontos de vista. A utilização de instrumento concebido em outro contexto sociocultural deve ser precedida de adaptação transcultural, envolvendo avaliação das equivalências semântica, de itens e conceitual. Vécu et Santé Perçue de l'Adolescent (VSP-A) é um instrumento genérico desenvolvido na França para avaliar a qualidade de vida relacionada à saúde de adolescentes. O objetivo deste trabalho foi verificar as equivalências semântica, de itens e conceitual da versão em português do VSP-A. Foram realizadas tradução e retradução do VSP-A, apreciação das equivalências, pré-teste e proposição de versão final a partir de discussão com especialistas. A edição em português do VSP-A mostrou ser semanticamente equivalente à original, apesar da necessidade de modificar alguns itens para facilitar seu uso. Resultados do pré-teste mostraram que o instrumento é facilmente compreendido e respondido pelos adolescentes. Para uso no Brasil, suas propriedades psicométricas necessitam ser avaliadas.

Adolescente; Qualidade de Vida; Estudos de Validação; Questionários

\section{Colaboradores}

M. T. Aires idealizou o estudo, selecionou o questionário a ser adaptado, coletou os dados, analisou os resultados e redigiu o manuscrito. G. L. Werneck idealizou o trabalho, discutiu a metodologia, analisou os resultados e redigiu o manuscrito.

\section{Agradecimentos}

Agradecemos aos autores do instrumento original, Marie-Claude Siméoni, Pascal Auquier e Grupo de Investigação de Qualidade de Vida da Faculdade de Medicina da Universidade do Mediterrâneo por orientação nas diversas etapas de adaptação do instrumento ao nosso idioma. Agradecemos aos Professores Antonio J. L. Costa, Arildo Franco, Luciane Guedes, Marcelo Land e Peter Liquornik pelas valiosas contribuições ao questionário. Somos gratos aos adolescentes e professores da escola participante.

\section{Referências}

1. Rajmil L, Herdman M, Fernandez-de-Sanmamed MJ, Detmar S, Bruil J, Ravens-Sieberer U, et al. Generic health-related quality of life instruments in children and adolescents: qualitative analysis of content. J Adolesc Health 2004; 34:37-45.

2. Klatchoian DA, Len CA, Terreri MT, Silva M, Itamoto C, Ciconelli RM, et al. Quality of life of children and adolescents from São Paulo: reliability and validity of the Brazilian version of the Pediatric Quality of Life Inventory version 4.0 generic core scales. J Pediatr (Rio J.) 2008; 84:308-15.

3. Guedes DP, Guedes JERP. Tradução, adaptação transcultural e propriedades psicométricas do KIDSCREEN-52 para a população brasileira. Rev Paul Pediatr 2011; 29:364-71.
4. Simeoni MC, Auquier P, Antoniotti S, Sapin C, San Marco JL. Validation of a French health-related quality of life instrument for adolescents: the VSP-A. Qual Life Res 2000; 9:393-403.

5. Sapin C, Simeoni MC, El Khammar M, Antoniotti S, Auquier P. Reliability and validity of the VSP-A, a health related quality of life instrument for ill and healthy adolescents. J Adolesc Health 2005; 36: 327-36.

6. Serra-Sutton V, Herdman M, Rajmil L, Santed R, Ferrer M, Siméoni MC, et al. Adaptación al español del cuestionario Vecú et Sante Perçue de l'Adolescent (VSP-A): una medida genérica de calidad de vida para adolescentes. Rev Esp Salud Pública 2002; 76:701-12. 
7. Valencia MLJ, Martínez MR, Garcia JC, Palácio J, Alarcón LF. Validez linguística del cuestionario Vécu et Santé Perçue de l'adolescent (VSP-A) en población adolescente colombiana. Análisis y Modificación de Conducta 2007; 33:103-33.

8. Serra-Sutton V, Rajmil L, Berra S, Herdman M, Aymerich M, Ferrer M, et al. Fiabilidad y validez del cuestionario de salud y calidad de vida para adolescentes Vecú et Santé Perçue de l'Adolescent (VSP-A). Aten Primaria 2006; 37:203-9.

9. Reichenheim ME, Moraes CL. Operacionalização de adaptação transcultural de instrumentos de aferição usados em epidemiologia. Rev Saúde Pública 2007 ; 41:665-73.
10. Aires MT, Auquier P, Robitail S, Werneck GL, Simeoni MC. Cross-cultural adaptation and psychometric properties of the Brazilian-Portuguese version of the VSP-A (Vecu et Sante Percue de l'Adolescent), health-related quality of life instrument for adolescents, in a healthy Brazilian population. BMC Pediatr 2011; 11:8.

Recebido em 26/Nov/2011

Versão final reapresentada em 18/Jul/2012

Aprovado em 23/Jul/2012 
